\title{
Congestion Control Framework for Real-Time Services in OFDMA-based Systems
}

\author{
E. O. Lucena, F. R. M. Lima, W. C. Freitas Jr and F. R. P. Cavalcanti
}

\begin{abstract}
In [1] the authors proposed a Quality of Service (QoS)-driven adaptive Congestion Control (CC) framework that can provide QoS guarantees for the Voice over IP (VoIP) service in mixed traffic scenarios in a single carrier system. The original framework is composed of three Radio Resource Management (RRM) algorithms: Admission Control (AC), Packet Scheduling (PSC) and Load Control (LC). In this work we generalize that framework to systems that employ a multicarrier access scheme such as Orthogonal Frequency Division Multiple Access (OFDMA). In OFDMA-based systems, there is flexibility to exploit the frequency dimension to achieve even better performance gains. In this work, we propose significant changes especially in the PSC functionality where radio resource allocation is performed. Using simulations, we evaluate the generalized $\mathrm{CC}$ framework in an OFDMA-based system with a traffic mix composed of sessions from a Real Time (RT) and a Non-Real Time (NRT) service. The results show that the generalized $\mathrm{CC}$ framework not only protects the QoS of sessions from the RT service but also imposes only a small performance degradation to the NRT service.
\end{abstract}

Keywords-Congestion Control, OFDMA, RT services

\section{INTRODUCTION}

The mobile networks are continuously evolving in order to support more users, to achieve higher data rates, and to provide new (multimedia) services. This constant evolution makes possible a scenario where mobile networks are able to compete with fixed (wired) networks for the broadband market.

Services and applications can be classified in terms of several aspects such as directionality (unidirectional or bi-directional), symmetry of the communications (symmetric or asymmetric), interactivity, number of parties and delivery requirements [2]. Specifically, when delivery requirement is concerned the services are categorized as Non-Real Time (NRT) or Real Time (RT). RT services require a short time response between the communicating parts. In general, RT services impose strict requirements regarding packet delay and jitter. Voice over IP (VoIP) and online games are examples of this class of service. On the other hand, NRT services do not have tight requirements concerning packet delay although high packet delays are unacceptable. The major constraint of NRT services is the information integrity, i.e., information loss is not tolerable.

In order to assure the desired Quality of Service (QoS) of telephony (RT service) Second Generation (2G) and Third Generation (3G) networks utilize circuit-switching

The authors are from Wireless Telecommunication Research Group - GTEL / Department of Teleinformatics - DETI / Federal University of Ceará - UFC. Emails: \{evilasio,rafaelm,walter,rodrigo\}@gtel.ufc.br. connections, i.e., resources are dedicated to the flows ${ }^{1}$ since the session initialization. However, in order to efficiently support multiple services on the same network the operators are upgrading their core network to the All-Internet Protocol (IP) concept. Among the advantages of an All-IP architecture we can mention the efficient support to mass-market usage of any IP-based service and reduced Operational Expenditure (OPEX) and Capital Expenditure (CAPEX). Despite the advantages of this architecture, the QoS guarantees of RT services is a challenging task. With All-IP, the resources are packet-switched, i.e., the resources are dynamically allocated to the flows according to the demands. One strategy to guarantee the desired QoS for RT services is the utilization of prioritization among services [3].

When the mobile networks operate in normal load the prioritization can be inserted in the scheduling process [4], [5], [6], [7]. However, there are some factors that can cause congestion situations (overload and/or outage), such as random behavior of external interference, different mobility profiles and geographical location of mobile terminals. These factors can cause variations in cell load and the QoS experienced by the users. In these conditions, the prioritization should be applied in a more broader range through Congestion Control (CC) algorithms. The work [1] proposed a QoS-driven adaptive $\mathrm{CC}$ framework that joins the functionalities of Packet Scheduling (PSC), Admission Control (AC) and Load Control (LC). The objective of that framework is to guarantee the QoS of VoIP flows. Results are shown for a case study considering a High Speed Downlink Packet Access (HSDPA) system.

The mentioned CC framework was designed for a network with a multiple access scheme based on Time Division Multiple Access (TDMA). Nevertheless, the candidate systems to Fourth Generation (4G), such as Long Term Evolution (LTE) and Worldwide Interoperability for Microwave Access (WiMAX), have adopted Orthogonal Frequency Division Multiple Access (OFDMA) as the multiple access technology for downlink. With OFDMA, the resource granularity is higher due to the availability of frequency chunks for resource allocation. Therefore, in this work we generalize the CC framework of [1] to be applied in OFDMA systems. Besides that, we provide a performance evaluation in a mixed service scenario. The remaining of this document is organized as follows: section II presents relevant aspects of the OFDMA technology; in section III we show the generalized CC framework; the performance evaluation of the generalized CC framework is shown in a case study with VoIP and World Wide

\footnotetext{
${ }^{1}$ A terminal can bear multiple service flows. However, without loss of generality, we consider that a terminal corresponds to a flow.
} 
Web (WWW) in section IV; and finally the main conclusions and perspectives are summarized in section $\mathrm{V}$.

\section{Orthogonal Frequency Division Multiple ACCESS}

OFDMA is a multiple access scheme based on Orthogonal Frequency Division Multiplexing (OFDM). OFDM is a transmission technology that has been utilized in wired and wireless communications. Asymmetric Digital Subscriber Line (ADSL) broadband access and power line communications are examples of applications of OFDM in wired systems. In wireless systems, the OFDM technology is utilized in IEEE $802.11 \mathrm{a} / \mathrm{g}$ and planned to be utilized in LTE and Mobile WiMAX.

In OFDM, the available frequency band for transmission is divided into several subcarriers that have narrower bandwidth than the channel coherence bandwidth, as in Frequency Division Multiplexing (FDM) systems. However, the subcarriers in OFDM are designed to be orthogonal among each other, which leads to higher spectral efficiency than FDM as it is illustrated in Fig. 1. The narrowband subcarriers also imply simplified equalization process because of the flat fading channel experienced in each subcarrier. Besides that, as the data rate transmitted in each subcarrier is low and consequently the modulated symbols are longer than the delay spreading, OFDM is robust against Inter Symbol Interference (ISI). In order to effectively mitigate the effects of ISI, a guard interval named cyclic prefix, that consists in a copy of part of the OFDM symbol, is inserted before the OFDM symbol transmission. More details about OFDM can be found in [8].



Fig. 1. Frequency-time representation of an OFDM signal [9]

With OFDMA [10], the multiple access is achieved by the assignment of different subcarriers or block of them to individual User Equipments (UEs) at different time periods. More specifically, in OFDMA the system resources can be arranged in a time-frequency grid as shown in Fig. 2. In the frequency axis the granularity is defined by the subcarriers while in the time dimension it is defined by an OFDM symbol.

One of the advantages of an OFDMA-based system is the opportunity to benefit from frequency and multiuser diversities. Frequency diversity means that it is unlikely that

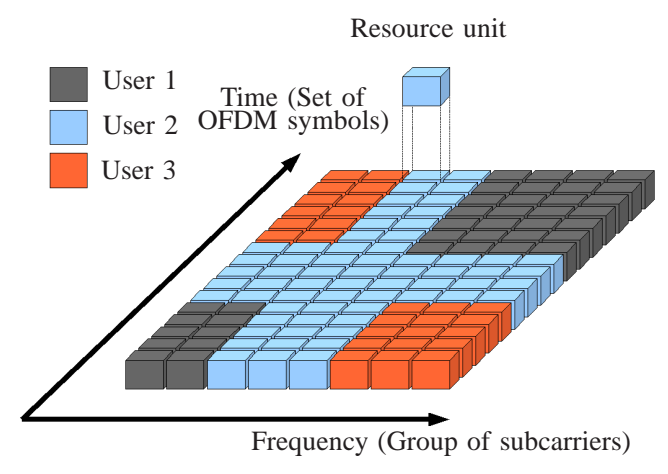

Fig. 2. Frequency-time resource grid in OFDMA.

all frequency resources in a link have the same channel quality. Multiuser diversity occurs due to the independence of UE channels caused by distinct UE positions in a cell, therefore, frequency resources in poor channel states for some UEs possibly will be in good channel conditions for other UEs. A mechanism for taking advantage of the frequency and multiuser diversities is the employment of scheduling algorithms. Scheduling algorithms are responsible for selecting which UEs will have access to the system resources and with which configuration. In this way, scheduling algorithms have a great impact on system performance.

\section{Congestion CONTROL Framework}

In this section we present the generalized $\mathrm{CC}$ framework for OFDMA systems. The $\mathrm{CC}$ framework comprises in a coordinated manner the operation of the AC, PSC and LC algorithms.

\section{A. Admission Control}

The AC algorithms are responsible for granting/denying the access of a new flow to the system. The criterion used for decision can be the availability of physical resources or the service quality of the connected flows. The AC algorithms are important tools to control the congestion in a system. By rejecting the new connections, the QoS of the already connected flows can be maintained. In this work, a Session Admission Control (SAC) scheme is employed to guarantee the quality of a single priority RT service in a mix with other services.

The SAC considers the delay as the resource to be shared among flows in the system. In order to do that, the packet delays of the high RT service are regularly measured and filtered. There are two admission thresholds depending on the service type: $D_{R T}^{t h}$ for the RT service and $D_{\text {Other }}^{\text {th }}$ for other low prioritized services. Therefore, when a new RT flow tries to access the system the SAC algorithm will check if the filtered delay of the RT service is greater or lower than the RT admission threshold. If greater, the new flow is rejected; if lower the flow is admitted. More details about SAC can be found in [1], [11]. The procedure is the same if the new flow is of an NRT service. 
According to the admission thresholds, a service can be more prioritized than the others. Although in the original proposal of SAC these admission thresholds are fixed, the main idea in the $\mathrm{CC}$ framework is to adapt them according to the congestion status of an RT service.

\section{B. Packet Scheduling}

The PSC algorithm used in the original proposal is the Weighted Proportional Fair (WPF) [3]. With WPF, the (single) scheduled flow is the one with higher piority defined as in the following

$$
p_{j}[k]=w_{j}[k] \cdot\left(\frac{r_{j}[k]}{t_{j}[k]}\right),
$$

where $p_{j}[k]$ is the priority of UE $j$ at Transmission Time Interval (TTI) $k, r_{j}[k]$ is the supported data rate of UE $j$ at TTI $k$ (according to the channel state) and $t_{j}[k]$ is the filtered data rate of UE $j$ at TTI $k$ that provides a history of the allocated data rates in the past. $w_{j}[k]$ represents a service-dependent weight of UE $j$ at TTI $k$. If the flow is from an RT session, $w_{j}[k]$ is set to $W_{R T}$. On the other hand, if the flow is from another service the priority is equal to $W_{\text {Other }}$. Therefore, by setting different values to these weights $\left(W_{R T}\right.$ and $\left.W_{O t h e r}\right)$ some prioritization among the services can be accomplished. As in the SAC scheme, these weights are fixed in the original proposal. The CC framework adapts these weights to control the congestion in the RT service.

In an OFDMA-based system, the frequency diversity can be exploited by adding another dimension in that prioritization. Therefore, we have adopted the Weighted Multicarrier Proportional Fair (WMPF) scheduler [12] that is a natural generalization of WPF. The prioritization in WMPF is given by

$$
p_{j, n}[k]=w_{j}[k] \cdot\left(\frac{r_{j, n}[k]}{t_{j}[k]}\right),
$$

where $p_{j, n}[k]$ is the priority of UE $j$ in subcarrier $n$ at TTI $k$ and $r_{j, n}[k]$ is the supported data rate of UE $j$ in subcarrier $n$ at TTI $k$ (according to the channel state of subcarrier $n$ ). From the priorities $p_{j, n}[k]$ we can build the priority matrix $\mathbf{P}$. The flow selection consists in assigning the pair flow-subcarriers corresponding to the largest entry in the priority matrix $\mathbf{P}$. In this way, multiple flows can be scheduled simultaneoulsy with pontentially higher data rates. The pseudo-code of WMPF is presented in Algorithm 1.

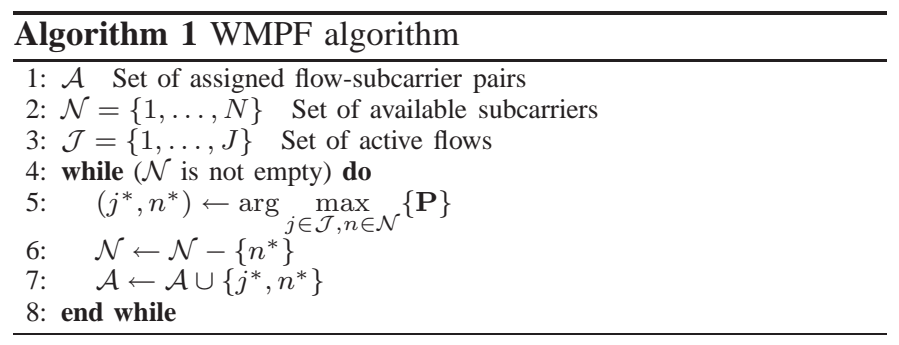

\section{Load Control}

Before describing the LC algorithm we should introduce two new variables: the SAC priority margin, $\alpha[k]$, and the WMPF priority margin $\beta[k]$. We define these priorities margins (in decibel) as

$$
\alpha[k]=10 \cdot \log _{10}\left(\frac{D_{\text {Other }}^{\text {th }}}{D_{R T}^{t h}}\right) \quad \beta[k]=10 \cdot \log _{10}\left(\frac{W_{\text {Other }}}{W_{R T}}\right),
$$

Considering that $D_{R T}^{t h}$ and $W_{R T}$ are fixed, the dynamic adaptation of the priority margins can control the prioritization of the RT service over the other services. The main idea of the LC algorithm is to adapt these priority margins according to the QoS of the ongoing sessions of the high priority RT service. If the quality of the sessions of this RT service is not being fulfilled, the LC algorithm decreases the priority margins. As a consequence, the sessions of this RT service will be scheduled more often and the system will decrease the number of admitted sessions of other services in order to protect the ongoing sessions of this RT service in the system.

The adaptation of the priority margin is calculated by means of a RT service measure. One example of RT service is the VoIP. As the VoIP quality can be measured by the Frame Erasure Rate (FER), the adaptation of the priority margin can be given as follows

$$
\alpha[k]=\min \left\{\max \left\{\alpha_{\min }, \alpha[k-1]-\sigma_{\alpha} \cdot e[k]\right\}, \alpha_{\max }\right\}
$$

$$
e[k]=F E R_{V o I P}^{f i l t}[k]-F E R_{V o I P}^{\text {target }} .
$$

The $\beta[k]$ is adapted in the same way as $\alpha[k]$ where $\beta_{\text {min }}$, $\beta_{\max }$ and $\sigma_{\beta}$ take place. $\alpha_{\min }, \alpha_{\max }, \beta_{\min }$ and $\beta_{\max }$ are the minimum and maximum values in $\mathrm{dB}$ of the $\alpha[k]$ and $\beta[k]$ parameters, respectively. $F E R_{V o I P}^{\text {filt }}$ is the filtered VoIP FER in the last control interval and FER $R_{V o I P}^{\text {target }}$ is the target VoIP FER to experience a good QoS. The fixed parameters $\sigma_{\alpha}$ and $\sigma_{\beta}$ control the speed of the priority margins $\alpha[k]$ and $\beta[k]$, respectively. The filtered FER $F E R_{V o I P}^{\text {filt }}[k]$ is obtained by appliyng a Simple Exponential Smoothing (SES) filter to the time series comprised by the average VoIP FER in each TTI.

From Equations (4) and (5) we can see that when the QoS of VoIP sessions in the system is worse than the expected $\left(F E R_{V o I P}^{f i l t}[k]>F E R_{V o I P}^{\text {target }}\right)$ the LC algorithm will decrease the priority margins. By decreasing the priority margins the VoIP sessions are prioritized in both AC and PSC algorithms. More details about the LC strategy see [1].

\section{Performance Evaluation}

In this section, we present a performance evaluation of our generalized CC framework. Details about the simulation tool and the main parameters used to obtain the results are presented in section IV-A. In section IV-B, we define the performance metrics used in this study and provide a description of the reference scenario used for comparison. Finally, in section IV-C we show and analyze the simulation results. 


\section{A. Simulations Parameters}

Our simulation tool models the main aspects of a single-cell OFDMA-based system. The simulation modeling includes aspects such as propagation phenomena, e.g., path loss, shadowing and fast fading; service applications and link adaptation.

We assume that the data symbols are independently modulated and transmitted over a high number of closely spaced orthogonal subcarriers. The modulation schemes Quadri-Phase Shift Keying (QPSK), 16 Quadrature Amplitude Modulation (QAM), and 64 QAM are available. The flows arrive in the system according to a Poisson distribution. Concerning traffic modeling we consider a mixed traffic scenario with VoIP as the RT service and WWW as the NRT service. The main parameters used for these simulations are presented in Table I.

TABLE I

MAIN PARAMETERS OF THE SIMULATION TOOL.

\begin{tabular}{|c|c|c|}
\hline Parameter & Value & Unit \\
\hline Cell radius & 500 & $\mathrm{~m}$ \\
\hline $\begin{array}{l}\text { Minimum distance } \\
\text { from Base Station (BS) }\end{array}$ & 100 & $\mathrm{~m}$ \\
\hline Maximum BS power & 5 & $\mathrm{~W}$ \\
\hline Number of Subcarriers & 200 & - \\
\hline Subcarrier spacing & 15 & $\mathrm{kHz}$ \\
\hline Carrier frequency & 2 & $\mathrm{GHz}$ \\
\hline UE speed & 3 & $\mathrm{~km} / \mathrm{h}$ \\
\hline Path loss [13] & $128+37.6 \cdot \log _{10}(d)$ & $\mathrm{dB}$ \\
\hline Shadow lognormal fading & $\sigma=8$ & $\mathrm{~dB}$ \\
\hline Small-scale fading & single-path Rayleigh & - \\
\hline Traffic mix (VoIP/WWW) & $50 / 50$ & $\%$ \\
\hline VoIP traffic model & according to [13] & - \\
\hline WWW traffic model & according to [14] & - \\
\hline $\begin{array}{l}\text { VoIP SAC delay threshold } \\
\left(D_{V o I P}^{t h}\right)\end{array}$ & 100 & $\mathrm{~ms}$ \\
\hline $\begin{array}{l}\text { VoIP WMPF priority weight } \\
\left(W_{V o I P}^{\text {prio }}\right)\end{array}$ & 1 & - \\
\hline Time basis for adaptation of $\alpha$ & 100 & $\mathrm{~ms}$ \\
\hline Time basis for adaptation of $\beta$ & 1 & $\mathrm{~ms}$ \\
\hline $\begin{array}{l}\text { Maximum value of } \alpha \text { and } \beta \\
\left(\alpha_{\max }, \beta_{\max }\right)\end{array}$ & 0 & $\mathrm{~dB}$ \\
\hline $\begin{array}{l}\text { Minimum value of } \alpha \text { and } \beta \\
\left(\alpha_{\min }, \beta_{\min }\right)\end{array}$ & -10 & $\mathrm{~dB}$ \\
\hline SAC step size $\left(\sigma_{\alpha}\right)$ & 0.5 & $\mathrm{~dB}$ \\
\hline WMPF step size $\left(\sigma_{\beta}\right)$ & 0.5 & $\mathrm{~dB}$ \\
\hline VoIP satisfaction threshold & 95 & $\%$ \\
\hline WWW satisfaction threshold & 90 & $\%$ \\
\hline VoIP FER threshold & 1 & $\%$ \\
\hline $\begin{array}{l}\text { WWW throughput } \\
\text { threshold }\end{array}$ & 64 & kbps \\
\hline
\end{tabular}

\section{B. Performance Metrics and Simulations Scenarios}

We compare the generalized CC framework to a reference scenario where no CC solution is applied, i.e., the WMPF and SAC priority margins are not adapted and are fixed with value equal to $0 d B$.

We define the offered load as the mean flow arrival rate (in number of flows per second) in the system. This is an input parameter to the Poisson processes used to model flow arrivals.
The blocking rate is the ratio between the number of new calls that were prevented from accessing the system and the total number of call attempts. The AC functionality has an important impact on this performance metric.

Regarding the WWW service, we consider the average throughput as the main QoS metric to assess the user satisfaction. During an interactive WWW session, a user may download several packet calls (WWW pages). We define the average throughput as the ratio between the size in bits of all the WWW pages downloaded during the lifetime of the flow and the activity time of the session.

The satisfaction level of the VoIP service is measured by the FER metric. The FER is the ratio of the number of lost packets (voice frames) and the total number of generated packets. The packet loss can occur for example when the packet is not transmitted before the deadline imposed by delay constraints.

Another important metric used in this study is the user satisfaction ratio. For a VoIP flow, the satisfaction is reached when it was not blocked by the AC functionality and the experienced FER is equal to or lower than 1\% [15]. For the WWW service, the flow is satisfied when it was not blocked and the average throughput is at least $64 \mathrm{kbps}$ [11].

Finally, we define the service capacity as the offered load in which the user satisfaction ratio is equal to the user satisfaction threshold. In this study we consider that the system operator defined the user satisfaction thresholds of VoIP and WWW services as $95 \%$ and $90 \%$, respectively.

\section{Results}

The result presented in Fig. 3 shows the blocking rate for both services. In the reference scenario, the blocking rate for VoIP and WWW services has almost a similar behavior once there is no service prioritization. When the adaptive CC framework is activated, we can see that the VoIP blocking rate decreases compared to the reference scenario. The reason for that is the higher priority of VoIP flows in the AC functionality achieved by the adjustment of SAC priority margin $(\alpha)$ performed by the generalized CC framework. From this figure we can also see that the blocking rate for WWW service with adaptive CC has a steep increase especially for higher loads. This degradation of WWW service is necessary to guarantee the QoS of VoIP flows even in overloaded conditions.

In Fig. 4 we show the behavior of the WWW throughput for two different loads. As expected, the higher is the offered load, the lower is the average throughput. Looking at the offered load of 1.38 , the average throughput provided by the adaptive $\mathrm{CC}$ framework and reference scenario are similar. However, at the load of 1.13 with CC activated we have around $30 \%$ of all users below the WWW throughput threshold (64kbps) instead of about $40 \%$ found in the reference scenario. This improvement in the average throughput with the adaptive CC framework is explained by the lower number of connected flows in the system due to the higher blocking rate imposed by the AC functionality. As there are fewer flows in the system, the flows in general can get more frequency resources.

In Fig. 5 we present the FER for two different loads. As expected, the VoIP FER increases with the system offered load, 


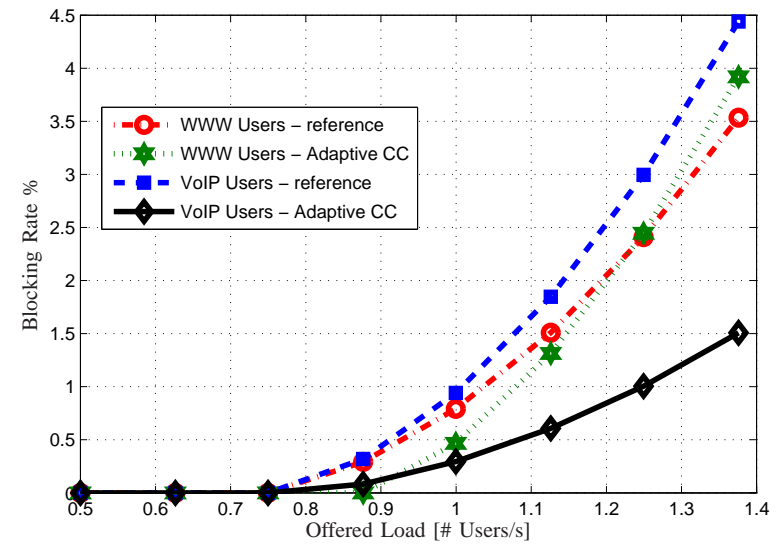

Fig. 3. Blocking Rate for Different Services with CC strategy and reference scenario.

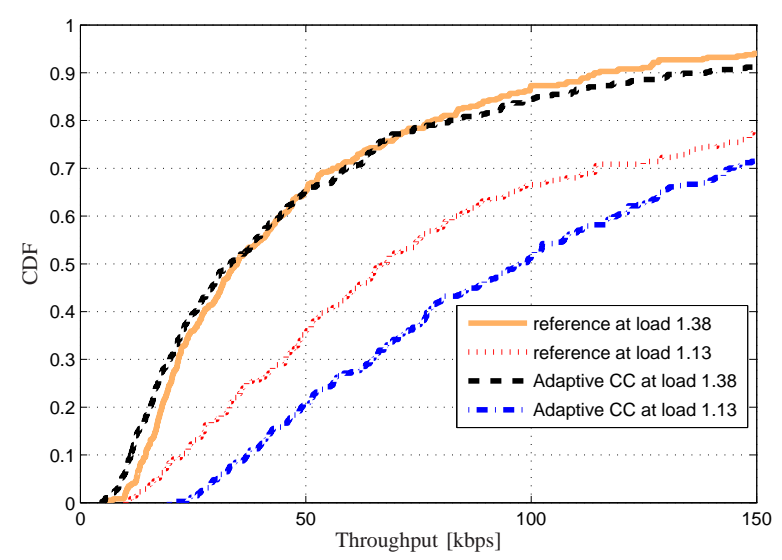

Fig. 4. CDF of Throughput of WWW users with CC strategy and reference scenario.

once in a overloaded system there are many packet losses due to unacceptable delays. We can observe that the VoIP FER is improved with the adaptive $\mathrm{CC}$ for the two loads presented if compared to the reference scenario. This shows that the generalized framework improves the QoS of the VoIP flows due to intelligent control of the SAC and WMPF priority margins based on the current VoIP quality.

In Fig. 6 we present the user satisfaction ratio for different loads. We can notice that user satisfaction for the WWW service with the adaptive $\mathrm{CC}$ framework and the reference scenario are similar. On the other hand, the user satisfaction for VoIP service is improved. Considering the user satisfaction threshold of $95 \%$ for VoIP we can observe a capacity gain of approximately $10 \%$. Therefore, our generalized CC framework improved the VoIP capacity with a smaller performance degradation for the WWW service.

\section{Conclusions And Perspectives}

In this article we adapt Congestion Control (CC) framework proposed by [1] to an Orthogonal Frequency Division Multiple Access (OFDMA)-based system. The original CC framework was designed to single-carrier systems and has the objective to protect the Quality of Service (QoS) of Voice over IP (VoIP) sessions in a mixed traffic scenario. The CC framework is composed of the following Radio Resource Management (RRM) functionalities: Admission Control (AC), Packet Scheduling (PSC) and Load Control (LC).

The $\mathrm{CC}$ framework is flexible in the sense that the adaptive Session Admission Control (SAC) and Weighted Multicarrier Proportional Fair (WMPF) schemes, the AC and PSC algorithms respectively, can be enabled in conjunction or separately. This may be interesting to cellular operators, who can suit this flexibility to their interests assessing aspects like complexity and performance trade-offs.

In OFDMA-based systems, there is a higher resource granularity and frequency diversity can be exploited by assigning different subcarriers to different users simultaneously. Therefore, in this work we propose significant changes in the PSC functionality of the CC framework.

We show simulation results where the generalized $\mathrm{CC}$ framework is applied in an OFDMA system with mixed traffic composed of VoIP and World Wide Web (WWW). The results show that the generalized $\mathrm{CC}$ framework not only protects

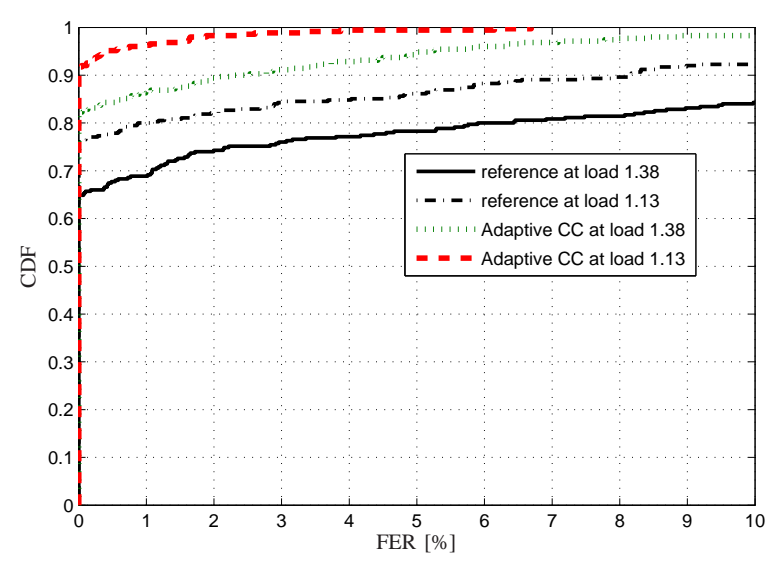

Fig. 5. FER CDF for VoIP users with CC strategy and reference scenario.

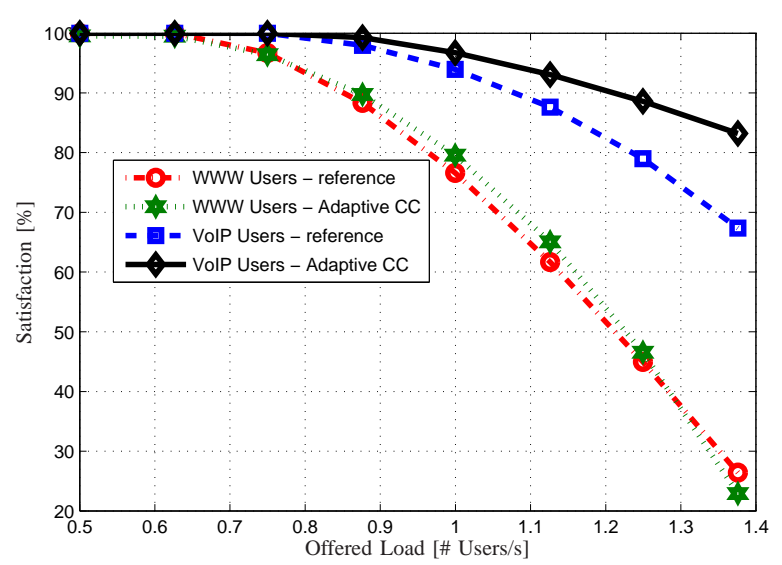

Fig. 6. User Satisfaction Ratio for Different Services with CC strategy and reference scenario. 
the QoS of the VoIP sessions but also imposes only a small performance degradation to the WWW.

As a perspective of this work we can mention the study of the generalized framework in other mixed traffic proportions between VoIP and WWW. Moreover, we plan to generalize this $\mathrm{CC}$ framework to scenarios where more than one service is prioritized.

\section{ACKNOWLEDGMENT}

This work was supported by the Research and Development Centre, Ericsson Telecomunicações S.A., Brazil, under EDB/UFC.22 Technical Cooperation Contract.

\section{REFERENCES}

[1] E. B. Rodrigues and F. R. P. Cavalcanti, "Qos-driven Adaptive Congestion Control for Voice over IP in Multiservice Wireless Cellular Networks," IEEE Communications Magazine, vol. 46, no. 1, pp. 100-107, Janeiro 2008.

[2] F. J. Velez and L. M. Correia, "Classification and Characterisation of Mobile Broadband Services," in Vehicular Technology Conference, 2000. IEEE VTS-Fall VTC 2000. 52nd, vol. 3, Setembro 2000, pp. 1417-1423.

[3] A. R. Braga, E. B. Rodrigues, and F. R. P. Cavalcanti, "Packet Scheduling for VoIP over HSDPA in Mixed Traffic Scenarios," in Personal, Indoor and Mobile Radio Communications, 2006 IEEE 17th International Symposium on, Helsinki, Setembro 2006, pp. 1-5.

[4] Y.-J. Choi and S. Bahk, "Scheduling for VoIP Service in CDMA2000 1x EV-DO," in Communications, 2004 IEEE International Conference on, vol. 3, Junho 2004, pp. 1495-1499.

[5] B. Chen, H. Hu, B. Wang, and H. Wang, "A Novel Multi-Service Scheduling Scheme for E-UTRA," in Internet, 2007. ICI 2007. 3rd IEEE/IFIP International Conference in Central Asia on, Setembro 2007, pp. 1-5.
[6] H. Lei, L. Zhang, X. Zhang, and D. Yang, "A Packet Scheduling Algorithm Using Utility Function for Mixed Services in the Downlink of OFDMA Systems," in Vehicular Technology Conference, 2007. VTC-2007 Fall. 2007 IEEE 66th, Outubro 2007, pp. 1664-1668.

[7] S. Choi, K. Jun, Y. Shin, S. Kang, and B. Choi, "MAC Scheduling Scheme for VoIP Traffic Service in 3G LTE," in Vehicular Technology Conference, 2007. VTC-2007 Fall. 2007 IEEE 66th, Outubro 2007, pp. 1441-1445.

[8] J. S. E. Dahlman, S. Parkvall and P. Beming, $3 G$ Evolution: HSPA and LTE for Mobile Broadband, 1st ed. Academic Press, Julho 2007.

[9] 3GPP, "Feasibility Study for Evolved Universal Terrestrial Radio Access (UTRA) and Evolved Universal Terrestrial Radio Access Network (E-UTRAN)," $3^{\text {rd }}$ Generation Partnership Project, Tech. Rep. TR 25.912 V7.2.0 - Release 7, Setembro 2006.

[10] M. Sternad, T. Svensson, T. Ottosson, A. Ahlen, A. Svensson, and A. Brunstrom, "Towards Systems Beyond 3G Based on Adaptive OFDMA Transmission," Proceedings of the IEEE, vol. 95, no. 12, pp. 2432-2455, Dezembro 2007.

[11] A. R. Braga, S. Wanstedt, and M. Ericson, "Admission Control for VoIP over HSDPA in a Mixed Traffic Scenario," in Telecommunications Symposium, 2006 International, Fortaleza, Ceara, Setembro 2006, pp. 71-76.

[12] Y. C. L. Yanhui, W. Chunming and T. Guangxin, "Downlink Scheduling and Radio Resource Allocation in Adaptive OFDMA Wireless Communication Systems for User-Individual QoS," in Transactions on Engineering, Computing and Technology - World Enformatika Society, no. 2, Março 2006, pp. 426-440.

[13] 3GPP, "Selection Procedures for the Choice of Radio Transmission Technologies of the UMTS," UMTS/ETSI, Tech. Rep. TR 101.112 v3.2.0, Abril 1998.

[14] C. Johansson, L. De Verdier, and F. Khan, "Performance of different scheduling strategies in a packet radio system," in Universal Personal Communications, 1998. ICUPC '98. IEEE 1998 International Conference on, vol. 1, Florence, Outubro 1998, pp. 267-271.

[15] 3GPP, "Performance Characterization of the Adaptive Multi-Rate Speech Codec," $3^{\text {rd }}$ Generation Partnership Project, Tech. Rep. TS 25.975 V6.0.0 - Release 6, Dezembro 2004. 